\title{
STRUCTURE CHANGES IN TRANSITION METAL OXIDES INDUCED DURING ELECTRON MICROSCOPY
}

\author{
D.S. Su, E. Zeitler, R. Schlögl \\ Department of Inorganic Chemistry, Fritz-Haber-Institute, Max-Planck Society, \\ Faradayweg 4-6, D-14195 Berlin, Germany
}

Many catalytic materials, especially the maximum valence transition metal oxides, are particularly susceptible to electron beam irradiation and thus undergo structural changes [1]. Hence knowledge about the behaviour of catalytic materials under the electron beam is of importance for all TEMinvestigations of such materials. On the other hand, this effect can be utilised for an in-situ study of the reductive property, phase transition and/or phase stability of various transition metal oxides in an inert, simple ambient high-vacuum [2]. The knowledge so obtained is needed for understanding the reduction mechanism of catalysts in more complicated chemical environments. In the present work, we study the electron beam induced change in $\mathrm{MoO}_{3}$ and $\mathrm{TiO}_{2}$ (anatase) by means of electron energy-loss spectroscopy (EELS), electron diffraction and high-resolution electron microscopy (HREM).

Molybdenum trioxide, $\mathrm{MoO}_{3}$, important as catalyst in the selective oxidation of hydrocarbons, forms an orthorhombic crystal layer structure. Fig. 1 shows oxygen $K$-edges recorded at various irradiation periods in a Philips 200 FEG electron microscope. The initial $\mathrm{O} K$-edge shows the features due to transitions of $\mathrm{O} 1 s$ electron to the co-valence mixed state derived from the $\mathrm{O} 2 p$ and Mo $4 d$ states. Due to the irradiation, the oxygen $K$-edge changes in shape and shifts to higher energy-losses, indicating a beam induced reduction of $\mathrm{MoO}_{3}$ of the irradiated area. Diffraction patterns and high-resolution imaging reveal the change of crystal lattice of $\mathrm{MoO}_{3}$ during the irradiation.

Titanium oxide is widely used as functional material in various field such as catalysis, photovoltaic and semiconductor devices. Fig.2A and 2B show the Ti $L$-edge and $\mathrm{O} K$-edge of anatase $\mathrm{TiO}_{2}$ recorded at various exposures to irradiation. The main feature of $\mathrm{Ti} L$-edges are the two doublet peaks arising from an electron transition from the Ti $2 p_{3 / 2}$ and $2 p_{1 / 2}$ levels to the Ti $3 d$ orbitals which split into $t_{2 g}$ and $e_{g}$ orbitals in the octahedral symmetry. These features remain unchanged after $20 \mathrm{~min}$ irradiation by a beam density of $3 \mathrm{~A} / \mathrm{cm}^{2}$; slight changes in shape of $L_{2}$ and $L_{3}$ edges, however, become visible after ca. 10 min radiation. The characteristics of the oxygen $K$ edge, the two peaks at 531 and $534 \mathrm{eV}$, are due to transitions of $\mathrm{O} 1 s$ electron to the co-valency mixed state derived from the $\mathrm{O} 2 p$ and $\mathrm{Ti} 3 d$ states. Irradiation does not change the shape of the edge, but rather shifts the peaks to slightly higher losses.

Acknowledgement

The work is supported by SFB 546 of the Deutsche Forschungsgemeinschft (DFG).

References

[1] R.J.D. Tilley and B.G. Hyde, J. Phys. Chem. Solids, Vol.21 (1970) 1613

[2] D.S. Su, M. Wieske, E. Beckmann, A. Blume, G. Mestl, R. Schlögl, submitted to Catalysis Letters (Dec. 2000) 


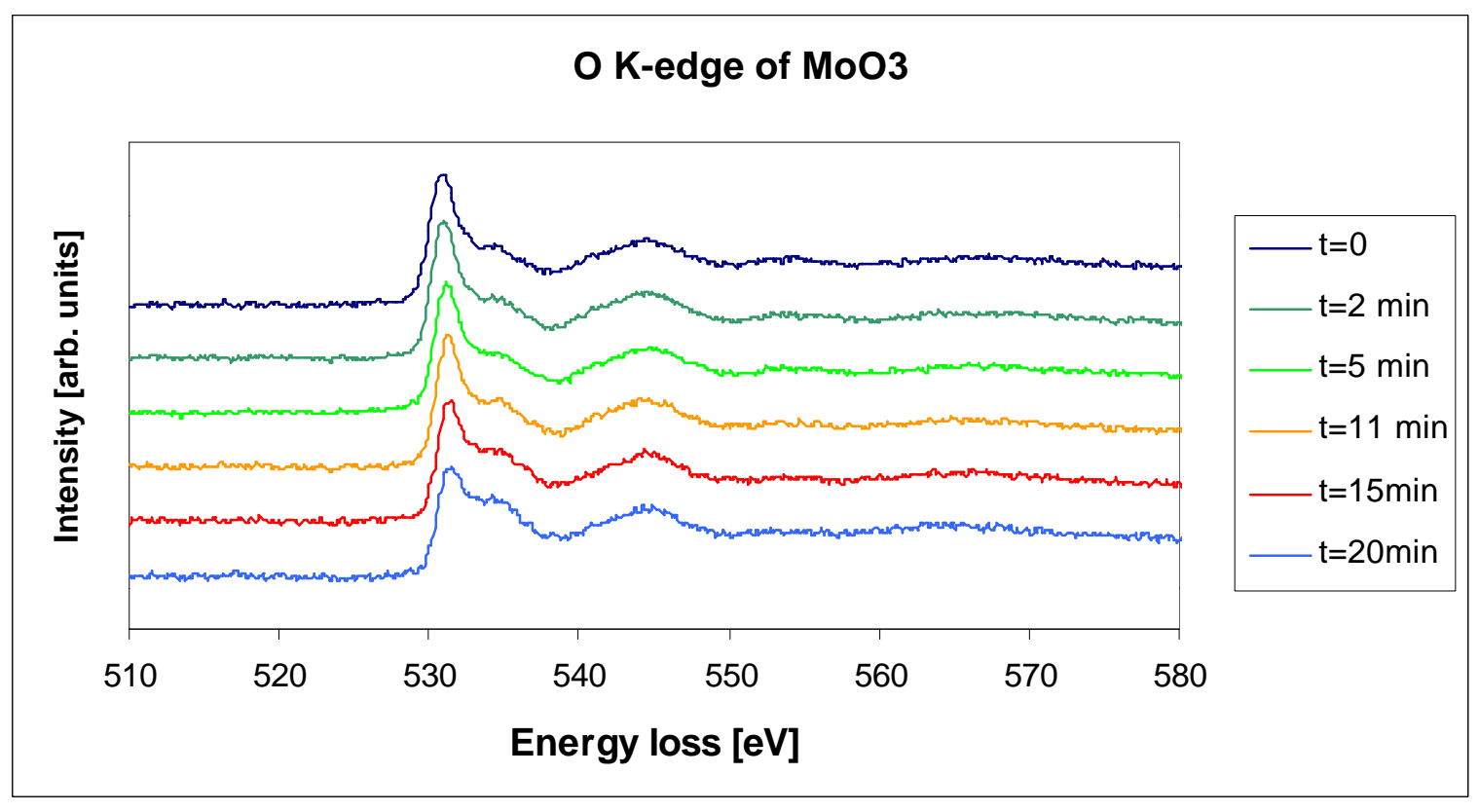

Fig.1 Changes in the oxygen $K$-edge of $\mathrm{MoO}_{3}$ after various periods of irradiation. Current density: $3 \mathrm{~A} / \mathrm{cm}^{2}$.
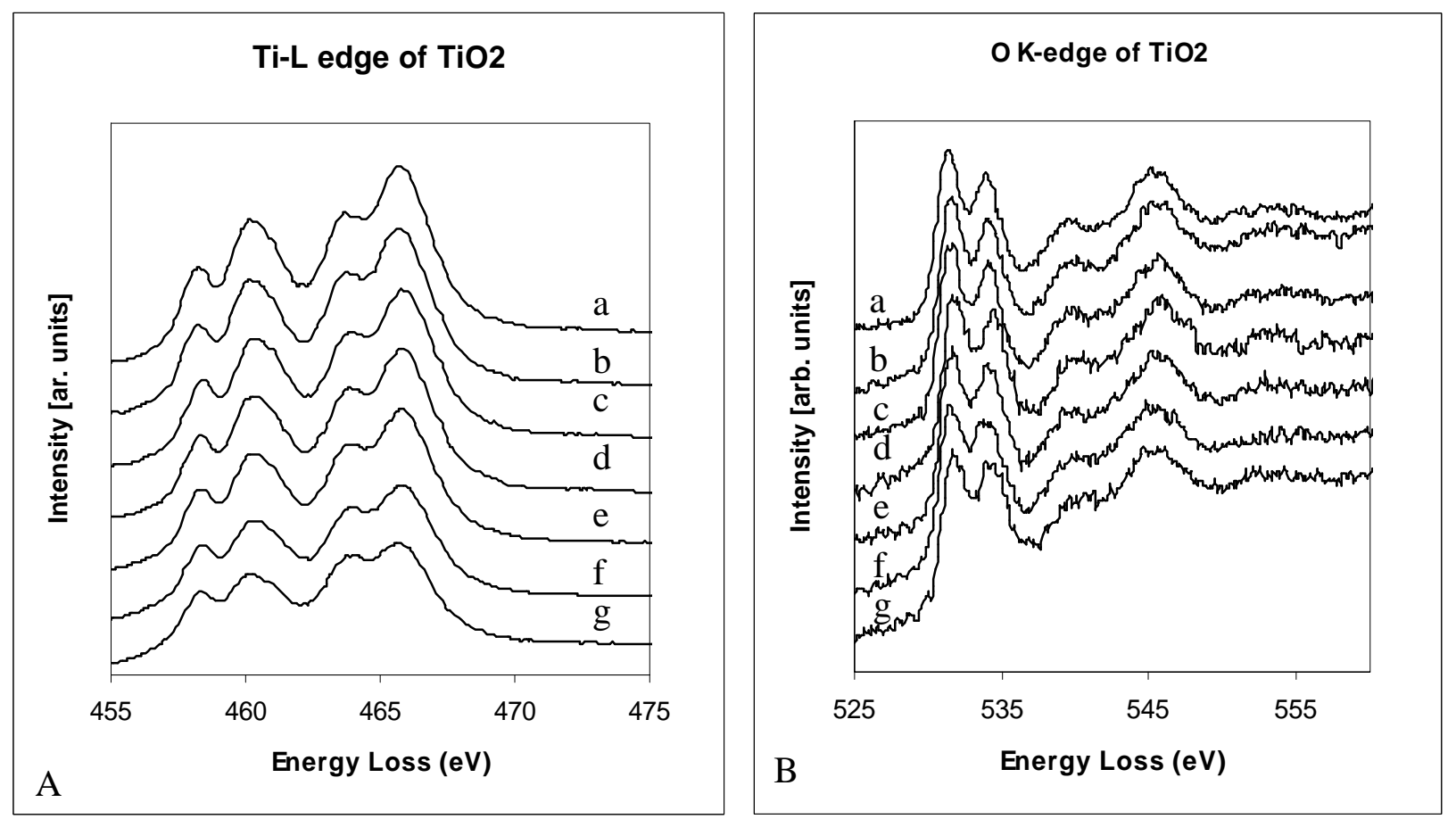

Fig.2 Changes in the Ti- $L_{2}, 3$ edges (A) and oxygen $K$-edge of $\mathrm{TiO}_{2}$ after various exposure times: a: beginning b: 3 min. c: 5 min. d: 7 min. e: 10 min. f: 15 min. g: $20 \mathrm{~min}$.

Current density: $3 \mathrm{~A} / \mathrm{cm}^{2}$. 